\title{
Sektorenübergreifende Versorgung und Vergütung
}

Martin Albrecht, Tamir Al-Abadi, Thomas Czihal und Sandra Mangiapane

13.1 Vergütungsunterschiede erschweren eine sektorenunabhängige Versorgung - 244

13.2 Ausmaß der Vergütungsunterschiede an der Sektorengrenze für ausgewählte Bereiche - 246

13.2.1 Auswahl der Fallbeispiele - 247

13.2.2 Datengrundlagen und Annahmen für die Kalkulation - 249

13.2.3 Ergebnisse - 250

13.2.4 Ursachen bestehender Vergütungsunterschiede - 252

13.3 Ansatzpunkte für sektorenunabhängige Vergütungsformen - 254

13.3.1 Ziele - 254

13.3.2 Abgrenzung relevanter Leistungsbereiche - 255

13.3.3 Maßstäbe für die Vergütungskalkulation - 256

$13.4 \quad$ Fazit -258

Literatur - 259 


\section{- Zusammenfassung}

Immer mehr Patientenbehandlungen können sowohl ambulant als auch stationär durchgeführt werden, werden aber je nach Behandlungsort sehr unterschiedlich vergütet. So beträgt die Vergütung der stationären Behandlung eines Patienten mit leichteren Herzrhythmusstörungen je nach Aufenthaltsdauer - das 2,6- bis 7-Fache derjenigen einer ambulanten Behandlung. Ursache hierfür ist die Existenz von zwei separaten Vergütungssystemen, die sich konzeptionell und in ihrer Systematik stark voneinander unterscheiden. Eine ungenügende Ausschöpfung des ambulanten Behandlungspotenzials und fehlende Leistungsgerechtigkeit der Vergütung gelten als Folge dieser sektoralen Trennung. In der gesundheitsökonomischen Diskussion wird daher zunehmend gefordert, die Vergütungen zu vereinheitlichen. Die Schaffung eines sektorenunabhängigen Vergütungssystems stößt jedoch auf eine ganze Reihe offener Fragen, die im vorliegenden Beitrag diskutiertwerden.

More and more patient treatments can be carried out both on an outpatient and inpatient basis, but are reimbursed very differently, depending on the place of treatment. Due to the existence of two separate reimbursement systems that differ greatly in concept and systematics, the reimbursement for, e.g., inpatient treatment of a patient with mild cardiac arrhythmias is 2.6 to 7 times higher than for outpatient treatment, depending on the length of stay. As a consequence of this sectoral separation, the exhaustion of the outpatient treatment potential is insufficient and the remuneration does not reflect performance equity. The health economic discussion is therefore increasingly calling for a standardisation of remuneration. However, the creation of a sector-independent remuneration system encounters a whole series of open questions which are discussed in this article.

\subsection{Vergütungsunterschiede erschweren eine sektorenunabhängige Versorgung}

Die mangelnde Integration der medizinischen Versorgung und die dadurch entstehenden "Schnittstellen“ zwischen den Leistungssektoren gelten als eine der zentralen Schwachstellen des deutschen Gesundheitssystems. Besonders große Effizienzverluste werden dabei in der Sektorierung der ambulanten und stationären Gesundheitsversorgung gesehen (SVRG 2012). Sektorierung bedeutet, dass für die ambulante Versorgung (durch Vertragsärzte) und die stationäre Versorgung (durch Krankenhäuser) strikt voneinander getrennte $\mathrm{Re}$ gelsysteme hinsichtlich der Kapazitätsplanung, Abrechnung und Vergütung, Dokumentation und Qualitätssicherung gelten. Infolge der seit vielen Jahren durch die Gesetzgebung erweiterte Öffnung der Krankenhäuser für die ambulante Versorgung - zuletzt beispielsweise in Form der Geriatrischen Institutsambulanzen, des Entlassmanagements und eines erweiterten Auftrags der Hochschulambulanzen - ist der "Schnittstellenbereich" zunehmend komplexer und unübersichtlicher geworden (Leber und Wasem 2016).

Dabei können zwei Arten der durch die Sektorierung entstehenden „Schnittstellenprobleme " unterschieden werden ${ }^{1}$ :

- Insbesondere bei älteren, chronisch bzw. mehrfach erkrankten Patienten, bei denen eine effektive Behandlung das Zusammenspiel mehrerer (ambulanter und stationärer) Leistungsanbieter voraussetzt, kann die Sektorierung zu Versorgungsbrüchen führen. $^{2}$ (SVR-G 2012)

1 Unterscheidung in Analogie zur Unterteilung sektorenübergreifender Verfahren in "sektorenüberschreitende" und "sektorgleiche" in der Richtlinie des G-BA zur datengestützten einrichtungsübergreifenden Qualitätssicherung.

2 Ein Beispiel für diesen Problembereich sind evtl. Versorgungslücken bei der weiteren medizinischen Betreuung nach einem Krankenhausaufenthalt. Um 
- Bei Leistungen, die prinzipiell sowohl ambulant als auch stationär erbracht werden können, beeinträchtigt die Sektorierung die Steuerung der Patienten in das unter Versorgungs- und Effizienzgesichtspunkten optimale Behandlungssetting.

Der folgende Beitrag konzentriert sich auf die Bedeutung der intersektoralen Vergütungsunterschiede für die Schnittstellenprobleme der zweitgenannten Art. Durch die erwähnte zunehmende Öffnung der Krankenhäuser für die ambulante Versorgung wurde dieser Leistungsbereich stark ausgeweitet. Hinzu kommt, dass infolge des medizinisch-technischen Fortschritts immer mehr Leistungen, die vormals nur stationär erbracht wurden, mittlerweile auch ambulant ausgeführt werden können. Somit hat die Anzahl der Leistungen zugenommen, die - obwohl therapeutisch gleichartig - wegen der sektoral separierten Vergütungssysteme je nach „Behandlungssetting“ unterschiedlich vergütet werden. Dies betrifft nicht nur die Höhe der Vergütung, sondern auch die ggf. damit verknüpften Mengenregulierungen.

Konkret werden vier Probleme diskutiert, die hieraus resultieren.

An erster Stelle steht die Kritik an einer zu geringen Ambulantisierung von Krankenhausleistungen. Neben dem Sachverständigenrat zur Begutachtung der Entwicklung im Gesundheitswesen (SVR-G 2012 und 2018) verweist hierauf auch die OECD regelmäßig. So liegt der Anteil potenziell vermeidbare Krankenhausfälle ausgewählter Indikationsbereiche $^{3}$ in Deutschland über dem EU-Durchschnitt und auch das Potenzial ambulante Operationen wird deutlich weniger ausgeschöpft als in anderen Ländern (OECD 2018). Zwar erhalten sowohl die niedergelassenen Ärzte als auch die Krankenhäuser für sog. AOP-Leistungen einheitliche Vergütungen nach dem EBM zu

solche Lücken zu vermeiden, wurde mit dem GKVVersorgungsstärkungsgesetz das Entlassmanagement nach $\S 39$ Abs. 1a SGB V neu geregelt.

3 Die OECD betrachtet hierbei die fünf Indikationen Diabetes, Bluthochdruck, Herzinsuffizienz, COPD und Asthma. denselben Konditionen (keine Mengenbegrenzung, nicht budgetiert). Allerdings kann ein Teil dieser Leistungen auch stationär erbracht werden, sodass der intersektorale Vergütungsunterschied letztlich relevant bleibt. ${ }^{45}$ So werden nach wie vor relevante Anteile von AOPLeistungen im Zusammenhang mit vollstationären Krankenhausfällen erbracht, die durch kurze Liegezeiten gekennzeichnet sind (Friedrich und Tillmanns 2016). Der Anteil der GKV-Ausgaben für im Krankenhaus ambulant durchgeführte Operationen stagniert seit Jahren und betrug zuletzt (2018) lediglich 0,8\% der gesamten GKV-Ausgaben für Krankenhausbehandlung. ${ }^{6}$

Speziell in der Hernienchirurgie ist der Anteil der ambulanten Operationen trotz einschlägiger Leitlinienempfehlungen im Vergleich zu anderen Ländern in Deutschland sehr gering (Koch et al. 2013; Steger et al. 2019). Als ein wesentlicher Grund hierfür werden die großen Vergütungsunterschiede zwischen ambulanter und stationärer Durchführung einer Leistenhernienoperation genannt.

Das nicht ausgeschöpfte Ambulantisierungspotenzial lässt sich darüber hinaus anhand der sog. ambulant-sensitiven Krankenhausfälle (ASK) kennzeichnen (vgl. Albrecht

4 Dies gilt für alle AOP-Leistungen, die in der Anlage zum AOP-Vertrag der Kategorie 2 zugeordnet werden (sowohl ambulant als auch stationär möglich), aber mit Begründung im Einzelfall auch für solche der Kategorie 1 (in der Regel ambulant).

5 Ähnlich ist die Konstellation in der Notfallversorgung: Krankenhäuser werden für die ambulante Notfallversorgung wie die Vertragsärzte im organisierten Notdienst nach EBM vergütet. Aus Sicht der Kliniken sind diese Vergütungen nicht kostendeckend (Haas et al. 2015). Diskutiert wird daher, dass hieraus Anreize zur Generierung unnötiger stationärer Fälle entstehen (Herr et al. 2018).

6 Außerdem gilt der AOP-Katalog als veraltet, sodass er die vermehrten Möglichkeiten für ambulante und stationsersetzende Behandlungen nicht mehr hinreichend abbildet (vgl. Gesetzentwurf MDK-Reformgesetz; $>$ https://www. bundesgesundheitsministerium.de/fileadmin/Dateien/ 3\protect_Downloads/Gesetzelprotect_und \protect_ Verordnungen/GuV/M/MDK-Reform \protect_Kabinett.pdf, Abruf: 14. August 2019). 
et al. 2014; Sundmacher et al. 2015). Sie bezeichnen Behandlungsfälle mit Erkrankungen, die zu großen Teilen als prinzipiell ambulant behandelbar gelten. Nach eigenen Berechnungen auf Basis der Krankenhausstatistik hat dieses Potenzial prinzipiell vermeidbarer Krankenhausfälle bundesweit eine Größenordnung von etwa 4 Mio., das entspricht einem Anteil von knapp $20 \%$ aller Krankenhausfälle (vgl. Loos et al. 2019). Gemäß dem Sachverständigenrat ist eine einheitliche Vergütungsstruktur die Voraussetzung dafür, dass der Grundsatz „ambulant vor stationär“, wie er in $\$ 39$ Abs. 1 Satz 2 SGB V gesetzlich verankert ist, konsequent umgesetzt wird (SVR-G 2018).

Ein zweites, damit verknüpftes Problem der intersektoralen Vergütungsunterschiede sind mögliche Verzerrungen der Wahl der Behandlungsmethoden. So zeigt sich am Beispiel der Leistenhernienchirurgie, dass bei stationärer Durchführung der operativen Behandlung überproportional häufig endoskopische Verfahren angewendet und doppelseitige Eingriffe durchgeführt werden, während im ambulanten Sektor offene Verfahren dominieren, ohne dass sich dies medizinisch ausreichend begründen ließe (Lorenz et al. 2015). Als mögliche Erklärungen hierfür wird angeführt, dass die Vergütungen ambulanter endoskopischer Leistenhernienoperationen nicht kostendeckend seien und dass bei Krankenhausaufenthalten ein endoskopisches Vorgehen bei der Frage der stationären Behandlungsbedürftigkeit von den Kostenträgern eher akzeptiert werde.

Grundsätzlich wird drittens kritisiert, dass es dem Kriterium der Leistungsgerechtigkeit widerspräche, wenn der Ort der Behandlung über die Höhe der Vergütung identischer ärztlicher Leistungen entscheide und nicht die Schwere der Erkrankung oder der ärztliche Aufwand (Bock et al. 2017). Allerdings kann der Ort der Behandlung durchaus leistungsrelevant sein, beispielsweise, wenn im Krankenhaus im Fall von unerwarteten Komplikationen eines Eingriffs schneller personelle oder apparative Unterstützung verfügbar ist. Werden entsprechende Vorhalteleistungen in Anspruch genommen, sind sie auch als preisrelevant zu berücksichtigen. Geschieht die Einbeziehung solcher Vorhalteaufwände in den Preis jedoch in intransparenter Weise, wird ein fairer Wettbewerb zwischen den Leistungsanbietern des ambulanten und des stationären Sektors erschwert.

Die sektoralen Vergütungsunterschiede werden zunehmend auch $\mathrm{zu}$ einem Problem für die Versorgung in ländlichen Regionen. Hier geht es weniger um einen fairen Wettbewerb zwischen Leistungsanbietern des ambulanten und stationären Sektors, sondern darum, den Zugang zu einer ambulanten und stationären Grundversorgung aufrechtzuerhalten. Letzteres wird in vielen Regionen unter den gegebenen wirtschaftlichen Rahmenbedingungen und den wachsenden Qualitätsanforderungen immer schwieriger. Als mögliche Lösung werden neue, sektorenübergreifende Organisationsformen und Leistungsangebote für solche Regionen diskutiert (z.B. Intersektorale Gesundheitszentren mit erweiterter ambulanter Versorgung, vgl. Schmid et al. 2018). Solche innovativen Versorgungsangebote lassen sich aktuell kaum auf Basis der regulären Vergütungssystematiken, sondern lediglich im Rahmen von Selektivverträgen (gemäß $\$ 140 \mathrm{a}$ SGB V) adäquat finanzieren.

Angesichts der aufgeführten Probleme wird ein neuer, einheitlicher Ordnungsrahmen für die ambulante fachärztliche Versorgung gefordert, der neben den Bereichen Bedarfsplanung, Qualitätssicherung und Innovationsregeln insbesondere auch die Vergütung umfasst (Leber und Wasem 2016; Malzahn und Jacobs 2016).

\subsection{Ausmaß der Vergütungsunterschiede an der Sektorengrenze für ausgewählte Bereiche}

Vor dem beschriebenen Hintergrund hat das IGES Institut in einer Studie für das Zentralinstitut für die kassenärztliche Versorgung in Deutschland ( $\mathrm{Zi})$ Vergütungsunterschiede an 
der Sektorengrenze anhand ausgewählter Fallbeispiele dargestellt (Albrecht und Al-Abadi 2018). Für den vorliegenden Beitrag wurden die Ergebnisse dieser Studie aktualisiert.

Bei den Fallbeispielen handelt es sich um Krankheitsbilder und/oder Behandlungsverfahren, die prinzipiell sowohl stationär als auch ambulant medizinisch versorgt werden können. Sie sollen exemplarisch veranschaulichen, wie groß die Vergütungs- bzw. Kostenunterschiede an der Sektorengrenze ausfallen, auf die sich die Forderung nach einer einheitlichen Vergütung bezieht. Einerseits werden damit die Herausforderungen verdeutlicht, die mit einer Umsetzung dieser Forderung verbunden wären, andererseits aber auch das Potenzial, durch eine - medizinisch vertretbare - Ambulantisierung der Versorgung an der Sektorengrenze Krankenhäuser und Beitragszahler zu entlasten.

Die Fallbeispiele stehen für typische Schnittstellenbereiche mit (quantitativer) Versorgungsrelevanz, sie erheben aber keinen Anspruch auf Vollständigkeit oder Repräsentativität und eignen sich daher auch nicht für eine Hochrechnung. Die ermittelten Vergütungsunterschiede lassen sich auch nicht vollständig als Einsparpotenziale interpretieren. ${ }^{7}$ Für die ausgewählten Fallbeispiele kann nämlich nicht in allen konkreten Situationen davon ausgegangen werden, dass eine stationäre durch eine ambulante Versorgung ersetzbar wäre - z. B. wenn eine soziale Indikation oder Erfordernisse einer interdisziplinären Versorgung dagegen sprechen.

\subsubsection{Auswahl der Fallbeispiele}

Um Vergütungsunterschiede an der ambulant-stationären Sektorengrenze zu ermitteln, sind Erkrankungsgruppen bzw. Behandlungsverfahren auszuwählen, die prinzipiell vollumfänglich in unterschiedlichen Settings der ambulanten und stationären Versorgung mit

7 Vgl. für einen solchen Ansatz am Beispiel der Schweiz Schwendener et al. (2016). gleichwertiger (Ergebnis-)Qualität behandelt werden können. Zu den unterschiedlichen Behandlungssettings zählen neben der vollstationären und der vertragsärztlichen Behandlung auch die ambulante Behandlung im und durch ein Krankenhaus sowie die belegärztliche Behandlung im Krankenhaus.

Den Ausgangspunkt für die Auswahl der Fallbeispiele bildet die Fallgruppenklassifikation des DRG-Systems, die zur Abgrenzung sowohl Diagnosen (Erkrankungen) als auch Prozeduren (Behandlungsleistungen) verwendet. Im Hinblick auf die sektorenübergreifende Relevanz wurden diejenigen Diagnosen/Prozeduren aus dem DRG-System ausgewählt, die durch eine relativ geringe mittlere Verweildauer sowie einen relativ niedrigen Schweregrad der dadurch erfassten Behandlungsfälle gekennzeichnet sind. Zusätzlich wurde bei der Auswahl die in der Fachliteratur verwendete Kategorie der ambulant-sensitiven Diagnosen berücksichtigt.

Die Auswahl orientierte sich darüber hinaus an drei weiteren Kriterien: Erstens wurde der Schwerpunkt auf Indikationen bzw. Leistungskomplexe gelegt, für die konservative (d.h. nicht operative) Behandlungen eine wesentliche Therapieoption darstellen und die daher für den ambulanten Sektor relevant sind. Die DRG-Auswahlgrundlage wurde zweitens auf versorgungsrelevante Leistungsgruppen begrenzt, d.h. auf die 100 der insg. rd. 1.300 DRG-Positionen mit der höchsten Abrechnungshäufigkeit. Schließlich stammen die Fallbeispiele aus dem Versorgungsspektrum verschiedener ärztlicher Fachrichtungen.

Auf Basis dieser Kriterien wurden insgesamt fünf DRGs ausgewählt: Tonsillektomie, Schlafapnoesyndrom bzw. Polysomnographie, Nicht schwere kardiale Arrhythmie, gastroenterologische Erkrankungen und Diabetes mellitus (-Tab. 13.1).

Für alle fünf Gruppen lag die Fallzahl (2017) jeweils bei über 80.000, die Anteile an der Gesamtfallzahl zwischen 0,4 und 2,6\% (die untere Grenze der 100 am häufigsten abgerechneten DRG-Positionen liegt bei einem Fallanteil von $0,24 \%)$. Die Anteile von Fällen mit 


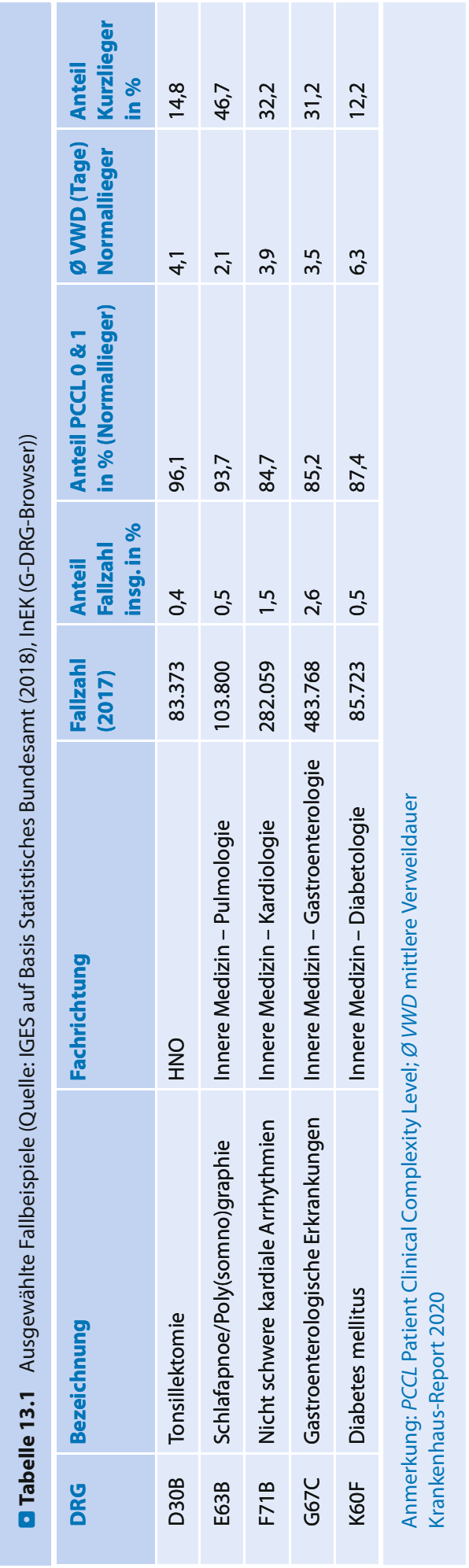

einem geringen Schweregrad (Patient Clinical Complexity Level von 0 oder 1) waren für die fünf ausgewählten Gruppen relativ hoch - bezogen auf die Anzahl der „Normallieger“ betrugen sie jeweils mehr als $80 \%$. Die mittlere Verweildauer lag in allen fünf Gruppen für Normallieger unter dem Durchschnitt aller Fälle von 7,3 Tagen (2017). Der Anteil der sog. Unter- bzw. Kurzlieger, also von Behandlungsfällen mit Unterschreitung der unteren Grenzverweildauer (jeweils zwei Tage), lag zwischen rd. $12 \%$ und rd. $47 \%$. Bis auf die kardialen Arrhythmien (F71B) umfassen alle ausgewählten Bereiche zudem Diagnosen, die zu den als ambulant-sensitiv eingestuften Diagnosen zählen.

Vier der fünf ausgewählten Fallbeispiele zählen zu unterschiedlichen Fachrichtungen der Inneren Medizin (Pulmologie, Kardiologie, Gastroenterologie, Diabetologie). Mit ihnen sind typischerweise nicht-operative Behandlungsverfahren assoziiert, die angesichts ihrer durchschnittlichen Verweildauer und Fallschwere prinzipiell sowohl für eine ambulante als auch für eine stationäre Versorgungsform infrage kommen. Als operatives Verfahren wurde die Tonsillektomie ausgewählt, die sowohl vollstationär als auch als belegärztliche Leistung im Krankenhaus durchgeführt werden kann. ${ }^{8}$ Die Tonsillektomie repräsentiert die Fachrichtung Hals-Nasen-Ohren-Heilkunde (HNO) und ist ein Beispiel für ein chirurgisches Fach.

\footnotetext{
8 Im Unterschied zur Tonsillektomie ist die operative Teilentfernung (Tonsillotomie) vergrößerter Gaumenmandeln (Hyperplasie der Tonsillen) seit einem Beschluss des Gemeinsamen Bundesausschusses (GBA) vom September 2018 auch ambulant im Krankenhaus oder in der vertragsärztlichen Versorgung durchführbar.
} 


\subsubsection{Datengrundlagen und Annahmen für die Kalkulation}

Dass die Behandlung von Patienten mit gleicher Indikation je nach Behandlungssetting unterschiedlich vergütet wird, ist auf die unterschiedlichen (sektorspezifischen) Vergütungssysteme zurückzuführen: das DRG-Fallpauschalensystem für stationäre Behandlungen, der Einheitliche Bewertungsmaßstab (EBM) für ambulante bzw. vertragsärztliche Leistungen und - im Fall belegärztlicher Leistungen - eine Kombination aus beidem. Diese Vergütungssysteme enthalten unterschiedliche Preise (Basisfallwerte vs. Punktwerte), vor allem aber verwenden sie ganz unterschiedliche Leistungsdefinitionen mit der Folge, dass einer Abrechnungsposition unterschiedlich (viele) Behandlungsprozeduren zugeordnet sind bzw. dieselbe Prozedur, je nach Art der Durchführung, zu unterschiedlichen Abrechnungspositionen führen kann (vgl. auch Hahn und Mussinghoff 2017). Für einen Vergütungsvergleich müssen daher den DRG-Fallpauschalen EBM-Positionen gegenübergestellt werden, die üblicherweise abgerechnet werden, wenn ein bezogen auf die DRG-Beschreibung typischer Fall ambulant behandelt wird.

Die Ermittlung der Vergütungsunterschiede basiert auf folgenden Datengrundlagen und Annahmen:

- Die Vergütungen der ausgewählten Indikationen bzw. Leistungskomplexe im stationären Sektor werden auf Basis des DRGKatalogs 2019 berechnet, d.h. die Bewertungsrelation der jeweiligen DRG wird auf einen Bundesbasisfallwert von 3.544,97€ angewendet. Dabei werden nur kurzstationäre Fälle mit einer Verweildauer von einem Tag (mit keiner oder einer Übernachtung im Krankenhaus) oder zwei Tagen (mit zwei Übernachtungen im Krankenhaus) betrachtet.

- Zu den so ermittelten DRG-Erlösen werden noch Mittel aus der KHG-Investitionsförderung für Krankenhäuser anteilig hinzugerechnet. Gemäß den Grundsätzen der dualen Finanzierung von Krankenhäusern gehen Investitionskosten nicht in die Kalkulation der Vergütung ein, stattdessen sind diese durch steuerfinanzierte Förderung der Bundesländer zu decken. Eine DRG-spezifische Zurechnung ist auf Basis der vom Institut für das Entgeltsystem im Krankenhaus (InEK) seit dem Jahr 2014 ermittelten Investitionsbewertungsrelationen möglich. Für die Fallbeispiele werden der Katalog der Investitionsbewertungsrelationen (IBR) zur G-DRG-Version 2019 sowie die mittleren Investitionskosten je Fall (IBR-Bezugsgröße, 2019: 339,90€) verwendet (InEK 2019a und 2019b). Auf der Grundlage der IBR-Bezugsgröße gelangen Schätzungen zu Investitionskosten bzw. einem bestandserhaltenden Investitionsbedarf in Höhe von rd. $6 \mathrm{Mrd}$. $€$ (vgl. z. B. Draheim und Beeck 2015). Da die tatsächliche KHG-Investitionsförderung der Bundesländer im Jahr 2017 nur knapp $2,8 \mathrm{Mrd}$. $€$ betrug, werden die DRG-spezifischen Investitionsbewertungsrelationen mit einem (fiktiven) Investitionsfallwert in Höhe von lediglich 50 \% der IBR-Bezugsgröße $(169,95 €)$ bewertet.

- Im Gegensatz zur Vergütung ambulanter Leistungen enthalten die DRG-Vergütungen Anteile zur Deckung von Kosten für Arzneimittel und Sachmittel. Diese sind für den Vergütungsvergleich zwischen DRG und EBM abzuziehen. Arzneimittelkosten finden in der vertragsärztlichen Vergütung gemäß dem EBM keine Berücksichtigung. Sachkosten werden nur in wenigen Fällen im EBM berücksichtigt und meist anderweitig gedeckt (z. B. im Rahmen von Verordnungen von Hilfsmitteln oder Sprechstundenbedarf). ${ }^{9}$

- Grundlage der Ermittlung der Vergütung für die ambulante Versorgung bildet der EBM für das Jahr 2019. Der für die Kal-

9 Entsprechend werden die DRG-Erlöse für den Vergleich um die Kostenarten 4a, 4b, 5, 6a-6c gemindert. Für eine detailliertere Beschreibung des Vorgehens vgl. Albrecht und Al-Abadi 2018. 
Tabelle 13.2 Fallbeispiel gastroenterologische Erkrankungen - häufigste stationäre Diagnosen und Prozeduren (2017) (Quelle: IGES auf Basis G-DRG-Browser 2017_2018 (InEK))

DRG G67C

\begin{tabular}{|l|l|}
\hline Fallzahl Anteil in \% \\
\hline (Normallieger)
\end{tabular}

\section{Hauptdiagnosen}

K59.0

$$
\text { Obstipation }
$$

A09.9

Sonstige und nicht näher bezeichnete Gastroenteritis und Kolitis

39.578

12,9

A09.0 nicht näher bezeichneten Ursprungs

\section{infektiösen Ursprungs}

Sonstige und nicht näher bezeichnete Gastroenteritis und Kolitis

K29.1 Sonstige akute Gastritis

39.463

12,9

$\mathrm{K} 21.0$

$$
\begin{aligned}
& \text { Gastroösophageale Refluxkrankheit mit Ösophagitis } \\
& \text { TOP } 5 \text { insg. }
\end{aligned}
$$

\begin{tabular}{l|l}
$19.643 \quad 6,4$
\end{tabular}

$169.989 \quad 55,7$

\section{Prozeduren}

\begin{tabular}{|l|l|c|c|}
\hline $1-632.0$ & Diagnostische Ösophagogastroduodenoskopie: Bei normalem Situs & 141.655 & 46,4 \\
\hline $1-440 . a$ & $\begin{array}{l}\text { Endoskopische Biopsie an oberem Verdauungstrakt, Gallengängen } \\
\text { und Pankreas: 1 bis 5 Biopsien am oberen Verdauungstrakt }\end{array}$ & 83.977 & 27,5 \\
\hline $1-650.2$ & Diagnostische Koloskopie: Total, mit lleoskopie & 48.311 & 15,8 \\
\hline $1-440.9$ & $\begin{array}{l}\text { Endoskopische Biopsie an oberem Verdauungstrakt, Gallengängen } \\
\text { und Pankreas: Stufenbiopsie am oberen Verdauungstrakt }\end{array}$ & 30.783 & 10,9 \\
\hline $3-225$ & Computertomographie des Abdomens mit Kontrastmittel & 25.787 & 8,5 \\
\hline Krankenhaus-Report 2020 & &
\end{tabular}

kulation verwendete Punktwert (Orientierungswert) beträgt damit 10,8226 Cent.

- Für die Auswahl der den DRGs gegenüberzustellenden EBM-Positionen wurde zweistufig vorgegangen: Zunächst wurde anhand der Daten des DRG-Browsers ermittelt, welche Diagnosen und welche Prozeduren für die ausgewählten DRGs mit der größten Häufigkeit dokumentiert und abgerechnet wurden. Für diese häufigsten Diagnosen haben Experten des Zi und der Kassenärztlichen Bundesvereinigung die in den vertragsärztlichen Abrechnungsdaten abgebildeten Leistungsketten und die diesbezüglich am häufigsten abgerechneten EBMZiffern nach inhaltlich-medizinischer Plausibilitätsprüfung ausgewählt. Für die altersdifferenzierte Zurechnung der Grundpau- schalen im EBM wurde die Altersstruktur der Behandlungsfälle gemäß den Daten des DRG-Browsers zugrunde gelegt.

\subsubsection{Ergebnisse}

Am Fallbeispiel der gastroenterologischen Erkrankungen (DRG G67C) wird das Vorgehen detaillierter beschrieben. Zunächst wurden für die ausgewählten DRG die häufigsten dokumentierten Hauptdiagnosen und Prozeduren ermittelt. Im Fall der DRG G67C handelt es sich um ein relativ breites Spektrum teilweise unspezifischer Diagnosen (•Tab. 13.2): Auf die fünf am häufigsten dokumentierten Hauptdiagnosen entfallen etwas mehr als die Hälfte aller Normallieger-Fälle. Dagegen ist das Spek- 


\begin{tabular}{|l|r|r|}
\hline \multicolumn{3}{|c|}{ Stationär (DRG) } \\
\hline DRG G67C (Haupt) & 1 Tag & 2 Tage \\
\hline + Investitionskosten & $67 €$ & $1.694 €$ \\
\hline - Arzneimittelkosten & $-34 €$ & $-34 €$ \\
\hline - Kosten für Sachmittel & $-148 €$ & $-148 €$ \\
\hline insgesamt & $671 €$ & $1.591 €$ \\
\hline
\end{tabular}

\begin{tabular}{|c|c|c|c|}
\hline \multicolumn{4}{|c|}{ Ambulant (EBM) } \\
\hline \multicolumn{2}{|c|}{ EBM-Ziffer } & \multirow{2}{*}{$\begin{array}{c}\text { Punkte } \\
191\end{array}$} & \multirow{2}{*}{$\begin{array}{r}\text { Euro } \\
20,71 €\end{array}$} \\
\hline 13342 & $\begin{array}{l}\text { gastroenterologische } \\
\text { Grundpauschale (altersgew.) }\end{array}$ & & \\
\hline 13400 & $\begin{array}{l}\text { Zusatzpauschale Ösophago- } \\
\text { Gastroduodenoskopie }\end{array}$ & 835 & $90,37 €$ \\
\hline 13401 & $\begin{array}{l}\text { Zusätzliche Leistung(en) im } \\
\text { Zus.hang mit GOP } 13400\end{array}$ & 513 & $55,52 €$ \\
\hline 13421 & Zusatzpauschale Koloskopie & 1.766 & $191,13 €$ \\
\hline 13422 & $\begin{array}{l}\text { Zusatzpauschale (Teil-) } \\
\text { Koloskopie }\end{array}$ & 1.080 & $116,88 €$ \\
\hline 13423 & $\begin{array}{l}\text { Zusätzliche Leistung(en) im } \\
\text { Zus.hang mit GOP } 13421 \\
\text { oder } 13422\end{array}$ & 262 & $28,36 €$ \\
\hline 19310 & $\begin{array}{l}\text { Histologische oder } \\
\text { zytologische Untersuchung } \\
\text { eines Materials }\end{array}$ & 83 & $8,98 €$ \\
\hline \multirow[t]{2}{*}{33042} & Abdominelle Sonographie & 157 & $16,99 €$ \\
\hline & insgesamt & & $528,94 €$ \\
\hline
\end{tabular}

Krankenhaus-Report 2020

- Abb. 13.1 Fallbeispiel gastroenterologische Erkrankungen - Vergütungsvergleich 2019 (Quelle: IGES auf Basis der InEK-Kalkulationsgrundlagen und des EBM)

trum der bei gastroenterologischen Erkrankungen im Krankenhaus zum Einsatz kommenden Prozeduren relativ stark konzentriert: Es dominieren diagnostische Maßnahmen bzw. Biopsien, in fast der Hälfte der Fälle wird eine Magenspiegelung durchgeführt.

Die Kalkulation der Vergütung gemäß dem beschriebenen Vorgehen (vgl. — Abschn. 13.2.2) ergibt für die stationäre Behandlung $671 €$ bei maximal einer Übernachtung und $1.591 €$ bei zwei Übernachtungen im Krankenhaus (- Abb. 13.1). Die Vergütung der ambulanten bzw. vertragsärztlichen Versorgung von gastroenterologischen Erkrankungen umfasst - orientiert an dem typischen Diagnose- und Prozedurenspektrum der stationären Versorgung - neben der gastroenterologischen Grundpauschale vor allem Zusatzpauschalen für die Ösophago-Gastroduodenoskopie und für (Teil-) Koloskopien. Zusammen mit weiteren Positionen summiert sich die kalkulierte Vergütung auf rd. $529 €$. Dabei ist zu beachten, dass bei der Berechnung der ambulanten Vergütung die möglichen relevanten Prozeduren - im Sinne einer konservativen Annahme - umfassend einkalkuliert wurden. Der resultierende Betrag deckt somit ein für den typischen Einzelfall tendenziell zu breites Leistungsspektrum ab.

Diesem Vorgehen entsprechend wurden die Vergütungsunterschiede auch für die vier anderen Fallbeispiele ermittelt (•Tab. 13.3). Die Ergebnisse zeigen, dass die Behandlung von Fällen, die hinsichtlich ihres Erkrankungsbildes bzw. dominierender Behandlungsmaßnahmen vergleichbar sind, bei stationärer Versorgung durchweg höher vergütet wird als in der ambulanten bzw. vertragsärztlichen Versorgung. Allerdings variieren die ermittelten Unterschiede zwischen den ausgewählten Bereichen erheblich: So reicht das Verhältnis der stationären zur ambulanten Vergütung vom 1,3-Fachen bis zum 10,3-Fachen. 
- Tabelle 13.3 Vergütungsunterschiede für ausgewählte Fallbeispiele im Überblick (2019) (Quelle: IGES auf Basis der InEK-Kalkulationsgrundlagen und des EBM)

\begin{tabular}{|c|c|c|c|c|c|c|}
\hline \multirow[t]{2}{*}{ DRG } & \multirow[t]{2}{*}{ Bezeichnung } & \multicolumn{2}{|c|}{ Vergütung stationär } & \multirow[t]{2}{*}{$\begin{array}{l}\text { Vergütung } \\
\text { ambulant }\end{array}$} & \multicolumn{2}{|c|}{$\begin{array}{l}\text { Verhältnis } \\
\text { stationär/ambulant }\end{array}$} \\
\hline & & 1 Tag & 2 Tage & & 1 Tag & 2 Tage \\
\hline \multirow[t]{3}{*}{ D30B } & Tonsillektomie & & & & ${ }^{*} 1,6$ & $* 1,6$ \\
\hline & - Vollstationär & $1.484 €$ & $2.608 €$ & & & \\
\hline & - Belegärztlich & $951 €$ & $1.678 €$ & & & \\
\hline E63B & Schlafapnoe/Poly(somno)graphie & $715 €$ & $1.058 €$ & $477 €$ & 1,5 & 2,2 \\
\hline F71B & Nicht schwerekardiale Arrhythmien & $578 €$ & $1.535 €$ & $220 €$ & 2,6 & 7,0 \\
\hline G67C & GastroenterologischeErkrankungen & $671 €$ & $1.591 €$ & $529 €$ & 1,3 & 3,0 \\
\hline K60F & Diabetes mellitus & $584 €$ & $2.443 €$ & $* * 236 €$ & 2,5 & 10,3 \\
\hline $\begin{array}{l}\text { Anmer } \\
\text { Koster } \\
\text { Kranke }\end{array}$ & $\begin{array}{l}\text { g: *Verhältnis vollstationär/belegä } \\
\text { er Patientenschulung (Annahme: } \\
\text { us-Report } 2020\end{array}$ & $\begin{array}{l}\text { tlich; *** } \\
\text { } €) \text {. }\end{array}$ & ation ar & & & usätzliche \\
\hline
\end{tabular}

Dabei stellt das zuvor detaillierter dargestellte Fallbeispiel der gastroenterologischen Erkrankungen den unteren Rand dieses Spektrums dar: Bei einem stationären Aufenthalt mit maximal einer Übernachtung übersteigt die kalkulierte stationäre Vergütung die ambulante Vergütung um $27 \%$. Die Vergütung der stationären Behandlung von Schlafapnoe übersteigt bei maximal einer Übernachtung die der ambulanten Behandlung bereits um $50 \%$. Deutlich größer sind die Unterschiede, wenn die stationäre Behandlung zwei Übernachtungen umfasst. Eine Ausnahme bildet das Fallbeispiel Tonsillektomie, bei dem die vollstationäre Behandlung mit einer belegärztlichen verglichen wird. Der Vergütungsabstand bleibt hier bei zwei Tagen Verweildauer in etwa gleich (bei rd. $55 \%)$.

Die höchsten Vergütungsunterschiede ergeben sich für die Fallbeispiele Nicht schwere kardiale Arrhythmien und Diabetes mellitus: Im ersten Fall betragen diese das 2,6-Fache (bei max. einer Übernachtung) bzw. das 7-Fache (bei zwei Übernachtungen). Für die kurzstationäre Versorgung von Fällen mit Diabetes mellitus liegt die ermittelte stationäre Vergütung bei max. einer Übernachtung um das 2,5-Fache über der ambulanten, bei zwei Übernachtungen sogar um das 10,3-Fache. Hierbei ist bereits berücksichtigt, dass Patientenschulungen - die häufigste Prozedur im Rahmen der DRG K60F - im EBM nicht abgebildet sind, dennoch aber auch ambulant durchgeführt werden. Vergütet werden diese dann im Rahmen von strukturierten Behandlungsprogrammen (DMP). Als Vergütungshöhe wurde für das Fallbeispiel ein Betrag von $100 €$ (inkl. Schulungsmaterial) angesetzt, der auf die EBM-Vergütung (136€) aufgeschlagen wurde. Die starke Veränderung des Vergütungsabstands im Fallbeispiel Diabetes mellitus ist darauf zurückzuführen, dass bei zwei Übernachtungen der Abschlag für das Unterschreiten der unteren Grenzverweildauer entfällt, der für „leichte Fälle“ vorgesehen ist.

\subsubsection{Ursachen bestehender Vergütungsunterschiede}

Konzepte für sektorenübergreifende bzw. sektorenunabhängige Vergütungsformen setzen das Verständnis der Rahmenbedingungen und Ursachen der bestehenden Vergütungsunter- 


\begin{tabular}{|c|c|c|}
\hline Vergütung & DRG & EBM \\
\hline \multirow[t]{2}{*}{ - Systematik } & \multicolumn{2}{|c|}{ Struktur- und Niveaukomponente } \\
\hline & Bewertungsrelationen und LBFW & Punktzahlen und Punktwert \\
\hline \multirow[t]{5}{*}{ - Kalkulation } & \multicolumn{2}{|c|}{ Prinzip der Vollkostenrechnung } \\
\hline & $\begin{array}{c}\text { Zuschlagskalkulation: Zuordnung } \\
\text { von Gemeinkosten zu } \\
\text { Fallgruppen als Kostenträger }\end{array}$ & $\begin{array}{c}\text { direkte Zurechnung der } \\
\text { Kostenarten (ärztl./techn.) zu } \\
\text { Einzelleistungen mit Zeitbedarf } \\
\text { (Ausn.: Versichertenpauschalen) }\end{array}$ \\
\hline & $\begin{array}{l}\text { Ist-Kosten } \\
\text { (Kalkulationshäuser) }\end{array}$ & $\begin{array}{c}\text { Soll-Kosten } \\
\text { (Schätzungen/normativ) }\end{array}$ \\
\hline & $\begin{array}{l}\text { regelmäßige umfassende } \\
\text { Erhebung/Anpassung }\end{array}$ & $\begin{array}{l}\text { nur partiell Erhebungen, } \\
\text { keine regelmäßige Überprüfung }\end{array}$ \\
\hline & InEK & BewA / InBA \\
\hline
\end{tabular}

- Abb. 13.2 Vergleich der Vergütungssysteme (Quelle: IGES)

schiede voraus. $\mathrm{Zu}$ den wesentlichen Unterschieden der ökonomischen Rahmenbedingungen zählt die deutlich größere Ressourcenbelastung des stationären Sektors durch räumliche, personelle (multidisziplinäre), apparative und zeitliche Kapazitätsvorhaltung (etwa im Rahmen der Notfallversorgung).

Mittelbar beeinflussen darüber hinaus Unterschiede zwischen stationärem und ambulantem Sektor im Hinblick auf die regulatorischen Rahmenbedingungen die Vergütungsund Kostenverhältnisse. Diese Unterschiede betreffen insbesondere die Kapazitätsplanung und - zumindest indirekt damit verknüpft die Ausgestaltung budget-begrenzender Maßnahmen, weiterhin Maßnahmen zur Qualitätssicherung und -förderung sowie den Umgang mit Innovationen (Erlaubnis- vs. Verbotsvorbehalt).

Die unmittelbaren Ursachen der dargestellten Vergütungsunterschiede liegen in den sektoral getrennten Vergütungssystemen für stationäre und ambulante Leistungen
(- Abb. 13.2). Dabei haben beide Systeme einige grundlegende Gemeinsamkeiten: Beide teilen die Vergütungssystematik in eine Struktur- und eine Niveaukomponente. Auch folgen beide Vergütungssysteme dem Prinzip der Vollkostenrechnung. Ansonsten überwiegen jedoch die Unterschiede in der Kalkulation der jeweiligen Vergütungshöhe:

- Die DRG-Vergütungen beruhen auf einer Zuschlagskalkulation auf Basis von Ist-Kosten (der Kalkulationshäuser) mit einer $\mathrm{Zu}$ ordnung der Gemeinkosten zu Fallgruppen als Kostenträger. Demgegenüber werden bei den EBM-Vergütungen unterschiedliche Kostenarten als Soll-Kosten direkt einzelnen Leistungen zugerechnet, deren Höhe sich u.a. an dem unterstellten Zeitbedarf orientiert. Die Soll-Höhe resultiert teilweise aus Schätzungen, teilweise aus normativen Setzungen. So wird beispielsweise der kalkulatorische Arztlohn, der einen relevanten Anteil der Vergütung ausmacht, normativ festgesetzt und dabei von einem selbststän- 
dig tätigen Arzt in eigener Praxis ausgegangen. Insgesamt hat dies u. a. zur Folge, dass im DRG-System auch vorgehaltene (nicht ausgelastete) Arbeitszeit in die Kalkulation der Vergütungshöhe einfließt, während gemäß EBM leistungsbezogene Norm-Arbeitszeiten vergütet werden.

- Die gesetzlichen Grundlagen enthalten für die Weiterentwicklung der Niveaukomponenten (Orientierungswert gem. $\$ 87$ Abs. 2e SGB V vs. Landesbasisfallwert/Veränderungswert gem. \$10 KHEntgG) unterschiedliche Vorgaben.

- Während die DRG-Vergütungen jährlich umfassend überprüft und angepasst werden, geschieht dies für EBM-Vergütungen anlassbezogen (siehe $\$ 87$ Abs. 2 SGB V) und nur partiell auf Basis von Erhebungen.

- Auch institutionell sind die Vergütungssysteme voneinander separiert: Für die Vergütung stationärer Leistungen sind die Selbstverwaltungspartner auf Bundesebene (Deutsche Krankenhausgesellschaft, Gesetzliche und Private Krankenversicherung) zuständig und das InEK übernimmt die wesentlichen Aufgaben im Zusammenhang mit der Weiterentwicklung und Pflege des G-DRG-Systems, für die vertragsärztliche Vergütung der Bewertungsausschuss Ärzte (aus Vertretern der Kassenärztlichen Bundesvereinigung und des GKV-Spitzenverbands), unterstützt durch das Institut des Bewertungsausschusses (InBA).

Ein weiterer wesentlicher Unterschied betrifft den Pauschalierungsgrad: Die DRG-Vergütungen umfassen prinzipiell mehrere (auch zeitlich stärker voneinander getrennte) Behandlungsschritte, die durch sie zu vergütenden Leistungsbündel sind jedoch nicht abschließend definiert. Der EBM gilt dagegen grundsätzlich als stärker einzelleistungsorientiert, er enthält mehr Positionen als das DRGSystem. ${ }^{10}$ Allerdings wurden im EBM in den vergangenen Jahren zuvor einzeln bewertete

10 Der EBM (Kapitel 1 bis 40) umfasst insgesamt 2.681 Gebührenpositionen (distinkte 5-Steller, Stand
Leistungen zu Komplexen und Pauschalen zusammengefasst, während der Pauschalierungsgrad im DRG-System durch die Einführung zahlreicher Zusatzentgelte sowie Zu-/Abschläge tendenziell konterkariert wurde. Auch die Art der Pauschalierung im EBM unterscheidet sich von der im DRG-System. So umfassen die hausärztlichen Versicherten- bzw. die fachärztlichen Grundpauschalen arztgruppentypische Grundleistungen für Patienten mit unterschiedlichen Diagnosen. Je nach Behandlungsfall können weitere Leistungen hinzukommen, die dann als Einzelleistungen abrechenbar sind. Im DRG-System werden dagegen möglichst kostenhomogene Fallgruppen anhand gleicher Diagnosen gebildet.

\subsection{Ansatzpunkte für sektorenunabhängige Vergütungsformen}

So wie die zunehmende Diskussion über die sektoral getrennten Vergütungssysteme verschiedene Probleme in den Blick nimmt darunter eine unzureichende Ambulantisierung und fehlende Leistungsgerechtigkeit (vgl. - Abschn. 13.1) -, so zeigt sich auch eine gewisse Vielfalt diskutierter Ansätze und Vorschläge für sektorenunabhängige Vergütungsformen. Diese unterscheiden sich hinsichtlich ihrer Ziele, der Abgrenzung der für eine sektorenunabhängige Vergütung relevanten Leistungsbereiche sowie hinsichtlich der Maßstäbe für die Höhe der Vergütung bzw. der Art ihrer Berechnung

\subsubsection{Ziele}

Als ein wesentliches Ziel sektorenunabhängiger Vergütung gilt die Schaffung gleicher Wettbewerbschancen (vgl. z. B. Malzahn und Jacobs 2016). Um Leistungsgerechtigkeit zu erreichen,

4. Quartal 2018). Im DRG-System (Katalog 2019) gibt es aktuell 1.313 Hauptabteilungs-DRGs. 
wird gefordert, die Vergütung konsequent am Leitprinzip ,gleicher Preis für gleiche Leistung“ auszurichten (Herr et al. 2018). Dagegen soll die Vergütung nicht mehr nach dem Ort bzw. dem konkreten Setting der Versorgung (Praxis, Ambulanz, Klinik) unterscheiden (vgl. Bock et al. 2017). Folgt man dieser Forderung, kann das konkrete Versorgungssetting (und damit u. U. auch die Vorhaltung personeller und/oder apparativer Leistungskapazitäten) nicht mehr expliziter Bestandteil der zu vergütenden Leistung sein.

Die wettbewerbliche Zielsetzung impliziert einerseits eine gewisse Ergebnisoffenheit bezüglich der Wahl des konkreten Versorgungssettings. So soll eine sektorenunabhängige Vergütung zu einer stärker patientenorientierten (Wahl der) Versorgung beitragen (den Patienten dort operieren, wo es für ihn am sinnvollsten ist, vgl. OPG 2018). Andererseits verbindet sich mit der Forderung nach (setting-)einheitlicher Vergütung häufig auch das Ziel, vollstationäre Krankenhausbehandlungen zu vermeiden bzw. gezielt Anreize zur Ambulantisierung bislang stationär erbrachter Leistungen zu erzeugen, um Einsparpotenziale zu erschließen (vgl. Malzahn und Jacobs 2016; SVR-G 2018; Bock et al. 2017).

\subsubsection{Abgrenzung relevanter Leistungsbereiche}

Die Entwicklung sektorenübergreifender Vergütungen setzt voraus, den Leistungsbereich mit einheitlicher Vergütung bei potenziell unterschiedlichen Behandlungssettings abzugrenzen von Leistungen, die ausschließlich ambulant oder stationär $\mathrm{zu}$ erbringen sind und weiterhin sektorspezifisch vergütet werden sollen. Hierzu gibt es unterschiedliche Vorstellungen und Konzepte.

Den Ausgangspunkt bilden häufig die ambulant durchführbaren Operationen (vgl. hier$\mathrm{zu}>$ Abschn. 13.1) und belegärztliche Leistungen. Umfangreicher ist der Leistungsbereich, der durch die ambulant-sensitiven Kran- kenhausfälle beschrieben wird (vgl. ebenfalls - Abschn. 13.1). Auch für die hier betrachteten Fallbeispiele wurden neben einer geringen Verweildauer und niedrigen Schweregraden ambulant-sensitive Diagnosen als ein Abgrenzungskriterium verwendet. Im Hinblick auf die erbrachten Leistungen für solche Fälle lässt sich auch die Kategorie sektorengleicher ${ }^{11}$ Behandlungsverfahren verwenden.

Ein bereits etablierter Ansatz mit einheitlicher Vergütung von Leistungen, die sowohl von Krankenhausärzten als auch von Vertragsärzten angeboten werden können, ist die ambulante spezialfachärztliche Versorgung (ASV). Die Abgrenzung dieses Bereichs folgt aber einer anderen Logik als die für die Fallbeispiele gewählte: Ausgangspunkt bilden hier nicht die „leichteren“ und damit potenziell ambulantisierbaren stationären Behandlungsfälle, sondern $\mathrm{Pa}$ tienten mit komplexen Krankheitsbildern, d. h. besonderen Krankheitsverläufen oder seltenen Erkrankungen sowie hochspezialisierte Leistungen - und zwar ausschließlich im Rahmen der ambulanten Behandlung.

Leber und Wasem (2016) diskutieren ein einheitliches Vergütungssystem begrenzt auf ambulante Klinikleistungen; dabei geht es ihnen vor allem auch um eine stärkere Differenzierung der Vergütungen. Sie plädieren für ein schrittweises Vorgehen, beginnend beispielsweise mit den Vergütungen von Leistungen der Hochschulambulanzen und psychiatrischen Institutsambulanzen, da die Einführung eines alle rechtlichen Formen ambulanter Krankenhausleistungen umfassenden Systems möglicherweise zu anspruchsvoll sei.

11 Gemäß der Richtlinie zur einrichtungs- und sektorenübergreifenden Qualitätssicherung des G-BA sind - im Unterschied zu sektorenüberschreitenden Verfahren - unter sektorengleichen Verfahren insbesondere solche zu verstehen, "die ein Thema betreffen, bei dem die Erbringung der gleichen medizinischen Leistungen in unterschiedlichen Sektoren erfolgt". Der Sachverständigenrat zur Begutachtung der Entwicklung im Gesundheitswesen bezeichnet sektorengleiche Verfahren als gleiche Behandlungen, die bei verschiedenen Patienten sowohl im ambulanten als auch im stationären Sektor erbracht werden können (SVR-G 2012). 
Bei Malzahn und Jacobs (2016) erstreckt sich hingegen die Forderung nach einer Vereinheitlichung der Vergütung auf die gesamte fachärztlich-ambulante Versorgung und somit auf alle daran teilnehmenden Ärzte und Krankenhäuser. Bock et al. (2017) sprechen sich explizit dagegen aus, den für notwendig erachteten einheitlichen Vergütungsrahmen nur auf den „Zwischenbereich“ zu beschränken, der durch divergierende Preise für (sektoren-)gleiche Leistungen gekennzeichnet ist. Stattdessen sollte sich der einheitliche Vergütungsrahmen auf den gesamten ärztlichen Bereich erstrecken, da die negativen Konsequenzen unterschiedlicher Vergütungsformen den gesamten stationären und ambulanten Leistungsbereich beträfen.

\subsubsection{Maßstäbe für die Vergütungskalkulation}

Unabhängig von der Abgrenzung ambulanter und stationärer Leistungen mit sektorenunabhängiger Vergütung bezieht sich die Diskussion über Art und Höhe der Vergütung häufig zunächst auf die Frage, ob eher das EBM- oder das DRG-System den Referenzrahmen bilden sollte. Dabei erscheinen die extremen Lösungen, wonach sektorengleiche Leistungen entweder einheitlich nach EBM oder einheitlich gemäß dem DRG-System vergütet werden, unrealistisch. Im ersten Fall wäre zumindest kurzfristig mit Kapazitätsproblemen zu rechnen, wenn Krankenhäuser die Versorgung der dann geringer vergüteten Fälle aufgeben, im zweiten Fall entstünden zusätzliche Ausgabenrisiken (Mengenexpansion).

Ein stärker differenzierender Ansatz wäre ein sektorenübergreifendes Tarifgeberprinzip, in Analogie zu den EBM-Vergütungen von Leistungen, die von verschiedenen Arztgruppen erbracht werden. Demnach würden Leistungskomplexe entweder nach EBM oder gemäß DRG vergütet. Die Zuordnung würde sich danach richten, welcher Sektor bzw. welches Behandlungssetting - gemessen z. B. am Fallanteil - leistungsprägend ist. Die Vergütung dieses leistungsprägenden Sektors/Behandlungssettings wäre dann maßgebend auch für die Anbieter des jeweils anderen Sektors/ anderer Behandlungssettings.

Alternativ kann für den Bereich sektorengleicher Leistungen eine eigenständige Vergütung entwickelt werden, die weder der heutigen ambulanten noch der stationären entspricht. Bislang gibt es hierfür allerdings wenig konkrete Anhaltspunkte. Impulse für die Entwicklung eigenständiger, sektorenübergreifender Kalkulationssystematiken der Vergütung könnten von der ASV ausgehen. Gemäß den gesetzlichen Vorgaben soll nämlich für die Vergütung in diesem Bereich auf betriebswirtschaftlicher Grundlage eine eigene Kalkulationssystematik mit diagnosebezogenen Gebührenpositionen entwickelt werden. Bislang liegen hierzu jedoch noch keine Ergebnisse vor. ${ }^{12}$

Der Entwurf des MDK-Reformgesetzes sieht die Schaffung eines aktualisierten und erweiterten AOP-Katalogs für stationsersetzende Behandlungen vor, um eine möglichst umfassende Ambulantisierung zu erreichen. Für die Leistungen dieses Katalogs sollen - erstmals bis Jahresende 2023 - für Krankenhäuser und Vertragsärzte einheitliche Vergütungen vereinbart werden. Die Vergütungen sind nach dem Schweregrad der Fälle zu differenzieren. Nach dem Referentenentwurf des Gesetzes sollten die Vertragsparteien die für Krankenhäuser und Vertragsärzte einheitlichen Vergütungen

12 Als Übergangslösung werden die im Rahmen der ASV abrechenbaren Leistungen, die vom G-BA als Behandlungsumfang festgelegt werden, gemäß EBM vergütet, allerdings ohne Anwendung mengen- bzw. budgetbegrenzender Maßnahmen und unmittelbar durch die Krankenkassen. Außerdem gilt für die ASV wie im stationären Sektor der Verbotsvorbehalt, sodass auch ambulante Leistungen erbracht werden können, die derzeit noch nicht durch den EBM abgebildet werden. Diese Leistungen werden nach der Gebührenordnung für Ärzte (GOÄ) vergütet (vgl. Jenschke et al. 2017). Für die Festlegung der ASV-Vergütung musste mit dem ergänzten Bewertungsausschuss (inkl. Arbeitsausschuss und Arbeitsgruppen) eine eigene Organisationsstruktur geschaffen werden (GKV-SV et al. 2017). 
„frei und unabhängig von einer bestimmten Vergütungsgrundlage vereinbaren“, gemäß dem Kabinettentwurf hingegen - analog zur ASV - auf betriebswirtschaftlicher Grundlage ausgehend vom EBM. Die Anknüpfung an einen bestehenden Vergütungsmaßstab soll verhindern, dass „mit der Vergütung der Katalogleistungen als sektorengleiche Leistungen ein dritter Sektor unkoordiniert neben dem stationären und dem ambulanten Sektor entsteht und neue Schnittstellenprobleme geschaffen werden. ${ }^{\text {"13 }}$

Entgegen dieser nun vorgesehenen EBMAnbindung wird in der gesundheitsökonomischen Diskussion häufiger eine Präferenz für eine DRG-Orientierung sektorenübergreifend vereinheitlichter Vergütungen geäußert. Leber und Wasem (2016) argumentieren mit Blick auf ambulante Krankenhausleistungen, eine Orientierung am EBM gehe in die falsche Richtung, denn dieser sei zu kleinteilig und zu sehr an einzelnen Leistungen sowie einzelnen Arztgruppen ausgerichtet und werde der für Krankenhäuser typischen Leistungserbringung im Team nicht gerecht. Bock et al. (2017) empfehlen, die Ausgestaltung eines neuen einheitlichen Vergütungssystems an DRG-Fallpauschalen zu orientieren, sodass Vergütungsunterschiede ausschließlich aus Patientencharakteristika bzw. indikationsbezogen resultieren (vgl. auch OPG 2018). Diese Vergütungsform eignet sich jedoch eher für Erkrankungen bzw. Behandlungen mit klaren Anfangs- und Endpunkten, also vor allem für operative und interventionelle Leistungen, weniger dagegen für chronische Erkrankungen oder die Ver-

13 Im Entwurf des MDK-Reformgesetzes heißt es weiter: „Dadurch bleibt eine gewisse Kongruenz zu den übrigen ambulanten Leistungen gewährleistet. Gleichwohl können die Vertragsparteien sachgerechte Vergütungen für Krankenhäuser und Vertragsärzte vereinbaren. So kann beispielsweise der im Durchschnitt entstehende Aufwand maßgeblich sein für die Festlegung der Vergütungen. Vor dem Hintergrund des neuen mit dem TSVG geschaffenen sektorenübergreifenden Schiedsgremiums ist zudem davon auszugehen, dass auch die Vergütungsinteressen der Krankenhäuser angemessen berücksichtigt werden." sorgung multimorbider Patienten (OPG 2018). Der hausärztliche Bereich bliebe weitgehend außerhalb des Geltungsbereichs eines neuen einheitlichen Vergütungssystems auf Basis von Fallpauschalen (Bock et al. 2017).

Neben der Vergütungsform, insbesondere Ausmaß und Art der Pauschalierung, stellen sich Fragen zur Höhe einer eigenständigen Vergütung für sektorengleiche Leistungen. Ein relativ einfacher Ansatz wäre, das Vergütungsniveau aus dem Mittel- bzw. Durchschnittswert der ambulanten und der stationären Vergütung zu bilden. So hat z. B. die Techniker Krankenkasse (TK) in einem Modellprojekt in Thüringen, bei dem für vier ausgewählte Behandlungskomplexe mit operativen Leistungen identische Vergütungen unabhängig vom Behandlungssetting gezahlt werden, sog. „Hybrid-DRGs“ gebildet. Für diese wurden Fallpauschalen als gewichtete Mittelwerte aus den Ausgaben der bisherigen ambulanten und stationären Leistungen bei gleichen Indikationen berechnet (OPG 2018).

Ein solches "mittleres Vergütungsniveau“ würde es für Krankenhäuser finanziell attraktiver machen, ambulant behandelbare Fälle auch ambulant zu behandeln. Gleichzeitig verringerten sich die Anreize für niedergelassene Ärztinnen und Ärzte, Patienten in Krankenhäuser einzuweisen, die u. U. auch weiter im ambulanten Setting behandelt werden könnten. Dass diese Anreize tatsächlich zum Tragen kommen, setzt jedoch voraus, dass eine umfassendere Reallokation von Behandlungsfällen an der Sektorengrenze nicht durch die Sektorierung der Honorar- bzw. Ausgabenbudgets sowie daran anknüpfende mengenregulierende Maßnahmen gehemmt wird.

Eine weitere Gestaltungsoption besteht darin, sich einem mittleren Vergütungsniveau schrittweise über einen längeren Zeitraum zu nähern. So könnte sich eine Mischvergütung zunächst eher am höheren DRG-Preisniveau orientieren, damit auch Krankenhäuser ambulante Leistungen kostendeckend anbieten können und nicht zu starke Anreize haben, stationäre Fälle zu generieren (Herr et al. 2018). Das Preisniveau könnte dann im Zeitverlauf abge- 
senkt werden. Die OECD verweist auf ein Vergütungsmodell in England, bei dem ambulant erbringbare Operationen bei tatsächlicher ambulanter Durchführung übergangsweise sogar höher vergütet werden als bei vollstationärer Durchführung, bis die ambulante Durchführung zur Norm geworden ist („Best Practice Tariffs for day surgery“, vgl. OECD 2018).

Eine aufwändigere Alternative zu Mischpreisen wäre eine Leistungsbewertung auf $\mathrm{Ba}$ sis einer Erhebung der tatsächlichen Kosten analog zur heutigen DRG-Kalkulation (und entsprechend mit periodischen Aktualisierungen der Datengrundlage). Die zentrale Frage hierbei ist, welches Behandlungssetting unter Effizienzgesichtspunkten die Referenz für eine solche Kostenerhebung sein soll. Während sich die Preisniveauentwicklung in der Krankenhausvergütung derzeit an der durchschnittlichen jährlichen Veränderung der Krankenhauskosten orientiert und damit die vorhandenen Strukturen abbildet, ${ }^{14}$ orientiert sich die Preisniveauentwicklung in der vertragsärztlichen Vergütung stärker normativ an den Kosten einer Einzelpraxis mit durchschnittlicher Leistungs- und Kostenstruktur (Herr et al. 2018). Die Forderung nach Kalkulationspraxen (Bock et al. 2017) für die Kalkulation sektorenunabhängiger Fallpauschalen bleibt daher unklar. ${ }^{15}$

Für sektorengleiche Leistungen stellt sich konkret die Frage, inwieweit einheitliche Ver-

14 Tatsächlich gilt für die Preisniveauentwicklung derzeit eine "Meistbegünstigungsklausel", wonach das Statistische Bundesamt auf Basis der Kostenstrukturen und -entwicklung der Krankenhäuser einen Orientierungswert für die Veränderung der Landesbasisfallwerte ermittelt. Dieser Orientierungswert sollte ursprünglich die sog. Grundlohnrate als Veränderungswert ablösen, letztere bleibt aber maßgeblich, wenn sie höher ausfällt als der Orientierungswert.

15 Auch der Sachverständigenrat schlägt die Gewinnung ambulanter Kalkulationspraxen vor, bezieht dies aber auf die Entwicklung von sektorenübergreifenden Leistungskomplexpauschalen für komplexe, chronische Erkrankungen, die alternierend sowohl ambulante als auch stationäre Episoden aufweisen und differenziert nach Schweregrad der Erkrankungen zeitraumbezogen zu vergüten wären (SVR-G 2018). gütungen von der Umlage stationärer Gemeinkosten befreit werden können und sollen. Beivers und Neubauer (2018) schlagen beispielsweise eigenständige teilstationäre DRGs vor, bei denen die Kosten um die Hotelkomponente der stationären Versorgung gemindert wären und die - analog zu den im Niveau abgesenkten Beleg-DRGs - einen eigenen Komplex im DRG-Vergütungssystem bilden sollten. Auch neuartige, intermediäre Versorgungsstrukturen - wie etwa das Konzept der erweiterten ambulanten Versorgung (mit der Möglichkeit einer 24-Stunden-Betreuung und -Unterbringung in Intersektoralen Gesundheitszentren) - begründen ein im Vergleich zur DRG-Vergütung abgesenktes Niveau (vgl. Schmid et al. 2018).

Alternativ ließe sich eine stärkere Unabhängigkeit der Leistungsvergütung vom konkreten Behandlungssetting durch eine Aufteilung der Vergütung in eine fallzahlabhängige und eine fallzahlunabhängige Komponente erreichen. Mit letzterer könnte in Form von pauschalen Zuschlägen Kostenstrukturunterschieden Rechnung getragen werden, die aus der Vorhaltung spezifischer Kapazitäten (z. B. IGZBetten) entstehen. ${ }^{16}$

Um die sektorenübergreifende Orientierung der Vergütungssysteme auch institutionell zu stärken, wird schließlich eine Fusion von InBA und InEK angeregt (vgl. Bock et al. 2017).

\subsection{Fazit}

In der Diskussion über eine stärker sektorenübergreifende Orientierung der Vergütungssysteme vermischen sich unterschiedliche Problemkreise und Argumentationslinien. $\mathrm{Zu}$ unterscheiden ist zunächst zwischen der sektorenüberschreitenden Versorgung von chronisch oder mehrfach erkrankten Patienten, bei der das Zusammenspiel mehrerer (ambulanter und

16 Der Sachverständigenrat diskutiert solche pauschalen Vergütungselemente für eine Reform der Betriebskostenfinanzierung von Krankenhäusern (SVRG 2018). 
stationärer) Leistungsanbieter im Vordergrund steht, und dem Bereich sektorengleicher Behandlungsverfahren, in dem es um die medizinisch optimale Wahl unter verschiedenen möglichen Behandlungssettings geht, aber auch um Wettbewerb und Kosten. Für letzteren zeigen die hier untersuchten Fallbeispiele ein teilweise beträchtliches Ausmaß von Vergütungsunterschieden, wenn Patienten mit vergleichbarer Indikation ambulant oder stationär behandelt werden.

Das so häufig geforderte Leitprinzip "gleicher Preis für gleiche Leistung“, d. h. eine Vergütung völlig unabhängig vom Behandlungsort/-setting, wird der Komplexität der Herausforderungen nicht gerecht, sektorenunabhängige Vergütungsformen zu entwickeln. In der gesundheitsökonomischen Diskussion wird zwar oft proklamiert, die Vergütung müsse vollständig unabhängig von den Versorgungsstrukturen sein und dürfe ausschließlich nach Patientencharakteristika bzw. Indikationen variieren; gleichzeitig aber werden von einem neuen Vergütungssystem Anreize gefordert, kostengünstigere ambulante Behandlungssettings systematisch zu bevorzugen (vgl. Bock et al. 2017).

Der Kern einer sektorenunabhängigen Vergütung besteht darin, den groben Dualismus von ambulant und stationär $\mathrm{zu}$ überwinden, d.h. die sehr unterschiedlichen Systeme zur Differenzierung von Vergütungen - primär einzelleistungs- und fachgruppenorientiert vs. diagnosebezogene, fachgruppenübergreifende und stärker pauschalierende Kostengruppen durch ein durchgängiges System der Differenzierung über die Sektorengrenze hinweg zu ersetzen. In einem solchen durchgängigen, sektorenübergreifenden System würde die Vergütungshöhe etwa nach dem Schweregrad von Erkrankungen und damit assoziierten Aufwandsunterschieden differenziert. Die Vereinheitlichung der Vergütung bedeutet also die Schaffung eines einheitlichen sektorenübergreifenden Differenzierungssystems, sodass die Vergütungshöhe in beiden Sektoren nach denselben, rein krankheits- bzw. patientenbezogenen Kriterien variiert. Ein solches System ist bislang ohne Beispiel.
Unterschiedliche Behandlungssettings und damit verbundene Kostenstrukturunterschiede sollten in einem solchen System durchaus weiterhin Berücksichtigung finden, allerdings könnte dies implizit geschehen, insoweit es durch eine höhere Vergütung bei einem höheren Schweregrad derselben Erkrankung ermöglicht, aufwändigere Behandlungssettings zu finanzieren. Aber auch hier bleibt die Frage nach dem (impliziten) Referenzsetting bestehen: Soll dieses rein empirisch und damit auf Basis bestehender Strukturen ermittelt oder normativ gesetzt werden? Und wie stark kann und soll hierbei pauschaliert bzw. differenziert werden?

Die Beantwortung dieser zentralen Gestaltungsfragen hängt schließlich auch davon ab, welche Veränderungen der Versorgungsstrukturen durch neue Vergütungsformen bewirkt werden sollen bzw. wie stark diese sein sollen. In diesem Zusammenhang werden häufig populationsorientierte regionale Budgets mit umfassender Abdeckung der ärztlichen Versorgung als eine Vergütungsform genannt, die sich mit der größten Konsequenz von konkreten Behandlungssettings löst und die Bildung innovativer Versorgungsstrukturen begünstigt. Mit einem solchen Ansatz überschreitet man allerdings die Grenze zwischen Leistungsvergütung und Versicherung, weil ein solches Budget letztlich die Risikoverteilung zwischen Kostenträgern und Leitungsanbietern verändert. Auch bleibt offen, nach welchen Maßstäben regionale Budgets unter den Leistungsanbietern verteilt werden sollen.

\section{Literatur}

Albrecht M, Al-Abadi T (2018) Perspektiven einer sektorenübergreifenden Vergütung ärztlicher Leistungen. Studie. Zentralinstitut für die kassenärztliche Versorgung in Deutschland, Berlin

Albrecht M, Schliwen A, Loos S (2014) Forschungsvorhaben zur Umsetzung des $\S 221$ b Abs. 2 SGB V Evaluierung der Auswirkungen von Zuschlägen zur Förderung der vertragsärztlichen Versorgung in unterversorgten Gebieten ( $\$ 87 a$ Abs. 2 Satz 3 SGB V) (Abschlussbericht) 
Beivers A, Neubauer G (2018) Hybrid-DRG - Die Richtung stimmt. F W Führen Wirtschaften Im Krankenh 2:154-158

Bock J-O, Focke K, Busse R (2017) Ein einheitliches Vergütungssystem für ambulante und stationäre ärztliche Leistungen - Notwendigkeit und Entwicklung. GuS 6:9-15

Draheim M, Beeck S (2015) Leistungsgerechte Finanzierung durch Investitionsbewertungsrelationen. Krankenhaus 9:818-822

Friedrich J, Tillmanns H (2016) Ambulante Operationen im Krankenhaus. In: Klauber J, Geraedts M, Friedrich J, Wasem J (Hrsg) Krankenhaus-Report 2016, Schwerpunkt: Ambulant im Krankenhaus. Schattauer, Stuttgart, S 127-147

GKV-Spitzenverband (GKV-SV), Deutsche Krankenhausgesellschaft (DKG), Kassenärztliche Bundesvereinigung (KBV) (2017) Bericht an das Bundesministerium für Gesundheit $\mathrm{zu}$ den Auswirkungen der ambulanten spezialfachärztlichen Versorgung auf die Kostenträger, die Leistungserbringer sowie die Patientenversorgung. https://www.gkv-spitzenverband.de/media/ dokumente/krankenversicherung_1/aerztliche_ versorgung/asv/2017-05-12_ASV_Bericht_ans_ BMG_Auswirkungen.pdf. Zugegriffen: 23. Aug. 2019

Haas C, Larbig M, Schöpke T et al (2015) Gutachten zur ambulanten Notfallversorgung im Krankenhaus - Fallkostenkalkulation und Strukturanalyse. https://www.dkgev.de/fileadmin/default/ Mediapool/2_Themen/2.2_Finanzierung_und_ Leistungskataloge/2.2.3._Ambulante_Verguetung/ 2.2.3.4._Ambulante_Notfallvehandlung_durch_ Krankenhaeuser/2015-02-17_Gutachten_zur_ ambulanten_Notfallversorgung_im_Krankenhaus_ 2015.pdf

Hahn U, Mussinghoff P (2017) Ökonomische Anreize belegärztlicher im Vergleich zu alternativen Versorgungsformen aus den Perspektiven von Krankenhaus und Vertragsarzt/Belegarzt sowie aus gesundheitssystemischer Sicht. Gesundheitsökonomie Qual 22:244-254

Herr D, Messerle R, Schreyögg J (2018) Status quo und gesundheitspolitischer Reformbedarf im ambulanten Vergütungssystem. GuS 4-5:8-15

Institut für das Entgeltsystem im Krankenhaus (InEK) (2019a) Abschlussbericht Entwicklung von Investitionsbewertungsrelationen (IBR) gem. § 10 KHG für das Jahr 2019. InEK, Siegburg

Institut für das Entgeltsystem im Krankenhaus (InEK) (2019b) Katalog der Investitionsbewertungsrelationen (IBR) zur G-DRG-Version 2019. InEK, Siegburg

Jenschke C, Munte A, Froschauer-Häfele S, Pöttgen S (2017) Sektorengrenzen ade? Analyse der Entwicklung der Versorgungsstrukturen in der ASV. In: Repschläger U, Schulte C, Osterkamp N (Hrsg) BARMER
Gesundheitswesen aktuell 2017. BARMER, Wuppertal, S 198-221

Koch A, Lorenz R, Meyer F, Weyhe D (2013) Leistenhernienreparation - Wo wird wie operiert? Zentralbl Chir 138:410-417

Leber W-D, Wasem J (2016) Ambulante Krankenhausleistungen - ein Überblick, eine Trendanalyse und einige ordnungspolitische Anmerkungen. In: Klauber J, Geraedts M, Friedrich J, Wasem J (Hrsg) Krankenhaus-Report 2016, Schwerpunkt: Ambulant im Krankenhaus. Schattauer, Stuttgart, S 3-28

Loos S, Albrecht M, Zich K (2019) Zukunftsfähige Krankenhausversorgung, Simulation und Analyse einer Neustrukturierung der Krankenhausversorgung am Beispiel einer Versorgungsregion in NordrheinWestfalen. Bertelsmann Stiftung, Gütersloh

Lorenz R, Koch A, Köckerling F (2015) Ambulante und stationäre Hernienchirurgie in Deutschland - aktueller Stand. Chir Allg 16(4):267-275

Malzahn J, Jacobs K (2016) Neuordnung der fachärztlichambulanten Versorgung. In: Klauber J, Geraedts M, Friedrich J, Wasem J (Hrsg) Krankenhaus-Report 2016, Schwerpunkt: Ambulant im Krankenhaus. Schattauer, Stuttgart, S 197-216

OECD, EU (2018) Health at a glance: Europe 2018: state of health in the EU cycle. OECD Publishing, Paris https:// doi.org/10.1787/health_glance_eur-2018-en

OPG (Operation Gesundheitswesen) (2018) Gesucht: Sektoral unvorbelastete Experten. Dr. Stephan Dittrich über die Erprobung der Hybrid-DRG, S 13-16

Sachverständigenrat zur Begutachtung der Entwicklung im Gesundheitswesen (SVR-G) (2012) Wettbewerb an der Schnittstelle zwischen ambulanter und stationärer Gesundheitsversorgung. Sondergutachten. Deutscher Bundestag Drucksache 17/10323

Sachverständigenrat zur Begutachtung der Entwicklung im Gesundheitswesen (SVR-G) (2018) Bedarfsgerechte Steuerung der Gesundheitsversorgung. Gutachten. Deutscher Bundestag Drucksache 19/3180

Schmid A, Hacker J, Rinsche F, Distler F (2018) Intersektorale Gesundheitszentren. Kassenärztliche Bundesvereinigung, Bayreuth (Gutachten)

Schwendener P, Sommer P et al (2016) Ambulant vor stationär. Oder wie sich eine Milliarde Franken jährlich einsparen lassen. https://www.pwc.ch/de/ publications/2016/ambulant_vor_stationaer_de_ 16_web_final.pdf. Zugegriffen: 23. Aug. 2019

Steger U, Bisping M, Urban J et al (2019) Minimalinvasive Leistenhernienchirurgie - TEP ambulant. Zentralbl Chir 144:26-31

Sundmacher L, Fischbach D, Schüttig W et al (2015) Which hospitalisations are ambulatory caresensitive, to what degree, and how could the rates be reduced? Results of a group consensus study in Germany. Health Policy 119(11):1415-1423. https:// doi.org/10.1016/j.healthpol.2015.08.007 
Open Access Dieses Kapitel wird unter der Creative Commons Namensnennung 4.0 International Lizenz (http:// creativecommons.org/licenses/by/4.0/deed.de) veröffentlicht, welche die Nutzung, Vervielfältigung, Bearbeitung, Verbreitung und Wiedergabe in jeglichem Medium und Format erlaubt, sofern Sie den/die ursprünglichen Autor(en) und die Quelle ordnungsgemäß nennen, einen Link zur Creative Commons Lizenz beifügen und angeben, ob Änderungen vorgenommen wurden.

Die in diesem Kapitel enthaltenen Bilder und sonstiges Drittmaterial unterliegen ebenfalls der genannten Creative Commons Lizenz, sofern sich aus der Abbildungslegende nichts anderes ergibt. Sofern das betreffende Material nicht unter der genannten Creative Commons Lizenz steht und die betreffende Handlung nicht nach gesetzlichen Vorschriften erlaubt ist, ist für die oben aufgeführten Weiterverwendungen des Materials die Einwilligung des jeweiligen Rechteinhabers einzuholen.

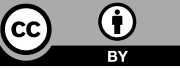

